\title{
An Efficiently Trained Deep Learning Potential for Graphane
}

\author{
Siddarth K. Achar, ${ }^{1}$ Linfeng Zhang, ${ }^{2}$ and J. Karl Johnson ${ }^{*}, 3$ \\ ${ }^{1)}$ Computational Modeling \& Simulation Program, University of Pittsburgh, Pittsburgh PA 15260, USA \\ ${ }^{2)}$ Program in Applied and Computational Mathematics, Princeton University, Princeton, NJ 08544, \\ United States \\ ${ }^{3)}$ Department of Chemical \& Petroleum Engineering, University of Pittsburgh, Pittsburgh PA 15261, USA
}

\section{Table of Contents}

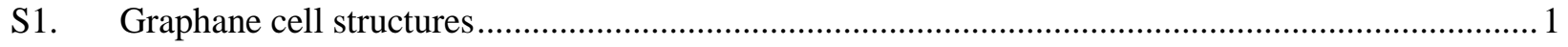

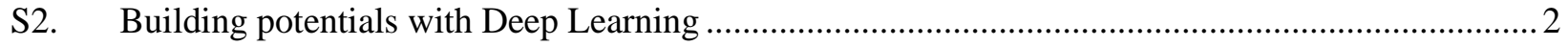

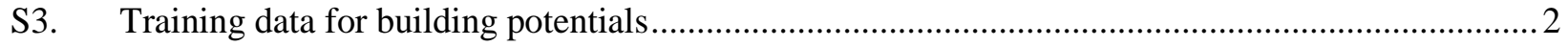

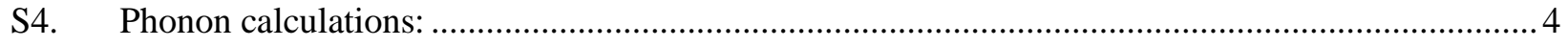

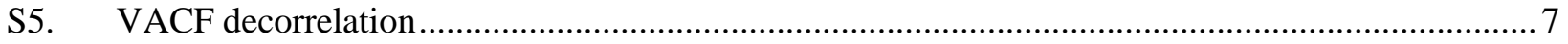

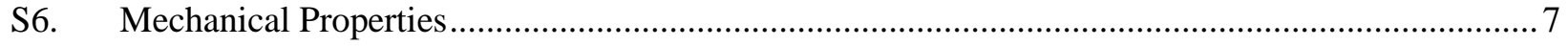

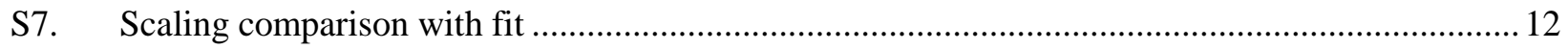

\section{S1. Graphane cell structures}

Table S1: Lattice parameters for cells of graphane used in the simulations.

\begin{tabular}{lcccccc}
\hline \# of Atoms & $\mathbf{a}(\mathbf{\AA})$ & $\mathbf{b}(\mathbf{\AA})$ & $\mathbf{c}(\mathbf{\AA})$ & $\boldsymbol{\alpha}$ & $\boldsymbol{\beta}$ & $\boldsymbol{\gamma}$ \\
\hline 80 & 12.69 & 8.88 & 20.00 & $90^{\circ}$ & $90^{\circ}$ & $90^{\circ}$ \\
160 & 25.39 & 8.88 & 20.00 & $90^{\circ}$ & $90^{\circ}$ & $90^{\circ}$ \\
320 & 25.39 & 17.77 & 20.00 & $90^{\circ}$ & $90^{\circ}$ & $90^{\circ}$ \\
480 & 38.08 & 17.77 & 20.00 & $90^{\circ}$ & $90^{\circ}$ & $90^{\circ}$ \\
8 (monoclinic) & 5.18 & 2.59 & 20.00 & $90^{\circ}$ & $90^{\circ}$ & $120^{\circ}$ \\
\hline
\end{tabular}




\section{S2. Building potentials with Deep Learning Deep-learning Potential (DP) training method}

The two atom types were carbon $(\mathrm{C})$ and hydrogen $(\mathrm{H})$ set in type map. There are several parameters set to define the descriptor $\mathrm{D}_{\mathrm{ij}}^{\alpha}$ to create an atomic chemical environment. The type of the descriptor was set to smooth-edition with full information (radial and angular). A cut-off radius of $5 \AA$ was chosen. The smoothing started at $4.8 \AA$. There were a maximum of $46 \mathrm{C}$ atoms and $92 \mathrm{H}$ atoms in the neighborhood within this cut-off. For the embedding net, the neuron sizes were set to 25, 50 and 100 (from input to output end) with the axis neurons of size 16. For the construction of the fitting net, the neuron size was set to 240, 240, 240 (from input to output) with a time step used in ResNet. ${ }^{1}$ An exponential learning rate was used for training the neural net with a decay rate of 0.95 . Training was then iteratively performed for $1,000,000$ batches to achieve higher accuracy and avoid overfitting of the training data.

\section{S3. Training data for building potentials Density Functional Theory Simulation Details}

To generate the training data for all the DP throughout the paper, density functional theory (DFT) molecular dynamics (MD) simulations were carried out using the Vienna Ab initio Simulation Package (VASP). ${ }^{2}$ Simulations were run for 1000 time steps with a time step of 0.5 fs. The size of graphane systems used in the simulations were varied to build different models. These different models were used for testing the effect of temperature and the effect of system size of graphane on the quality of predictions.

Projector augmented-wave $(\mathrm{PAW})^{3}$ pseudopotentials were used to describe valence electrons and frozen core interactions. An energy cut off of $400 \mathrm{eV}$ was used and the total energy convergence for self-consistent field calculations was set to $10^{-4} \mathrm{eV}$. A Monkhorst-Pack k-point grid size of 1 $\times 1 \times 1$ was used to perform the Brillouin zone sampling. All MD simulations were run in the $N V T$ (canonical ensemble) employing the Nosé-Hoover thermostat. ${ }^{4}$ The temperature of the thermostat was fixed to $1000 \mathrm{~K}$ (for most simulations) and $300 \mathrm{~K}$ (for comparison purposes).

We have tested both the DPs trained at $300 \mathrm{~K}$ and $1000 \mathrm{~K}$ to assess how well these two potentials extrapolate to temperatures beyond $1000 \mathrm{~K}$. We ran NVT DFT-MD simulations for 2.5 ps at $1250,1500,1750$ and $2000 \mathrm{~K}$ to generate test sets. Comparisons of root mean squared errors (RMSE) in predictions of the forces from both DPs are plotted in Figure S1. A linear fit to both these results shows that the rate of increase in RMSE for the $300 \mathrm{~K} \mathrm{DP}$ is 3.3 times that of the $1000 \mathrm{~K} \mathrm{DP}$. 


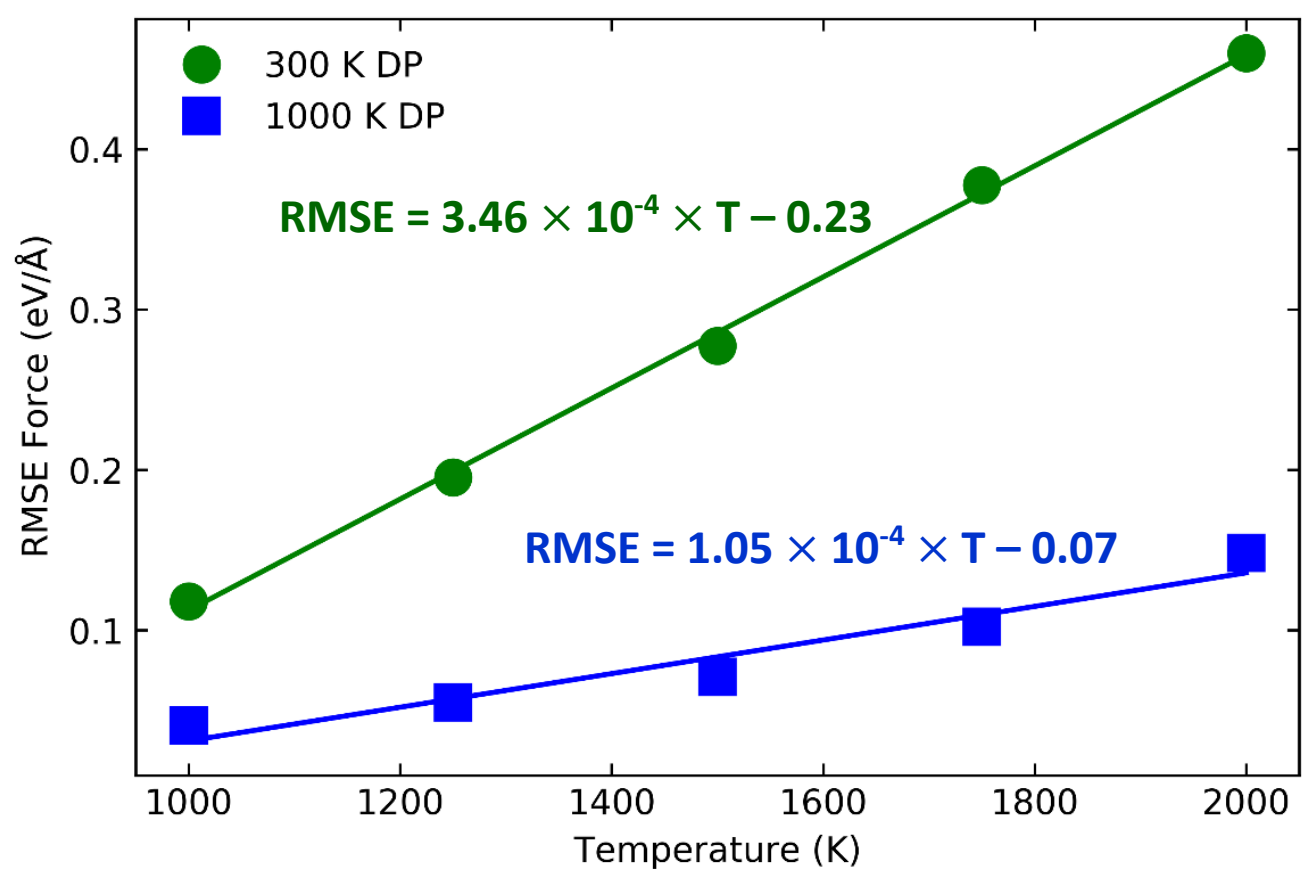

Figure S1: RMSE in the prediction of forces as a function of temperature for DPs trained at $300 \mathrm{~K}$ (green circles) and $1000 \mathrm{~K}$ (blue squares). The test set includes DFT-MD simulations from $1000 \mathrm{~K}$ to $2000 \mathrm{~K}$. Corresponding linear fits are plotted as straight lines long with the equation of fit above each plot.

We showed in the main text of the paper that energies and forces predicted by the $D_{80}$ and $D_{480}$ models are essentially identical. As a more stringent test of the accuracy of $D_{80}$ compared with $D_{480}$, we compared the phonon density of states (PDOS) computed from these potentials with that computed from VASP. From Figure S1, it is observed that there is no significant difference

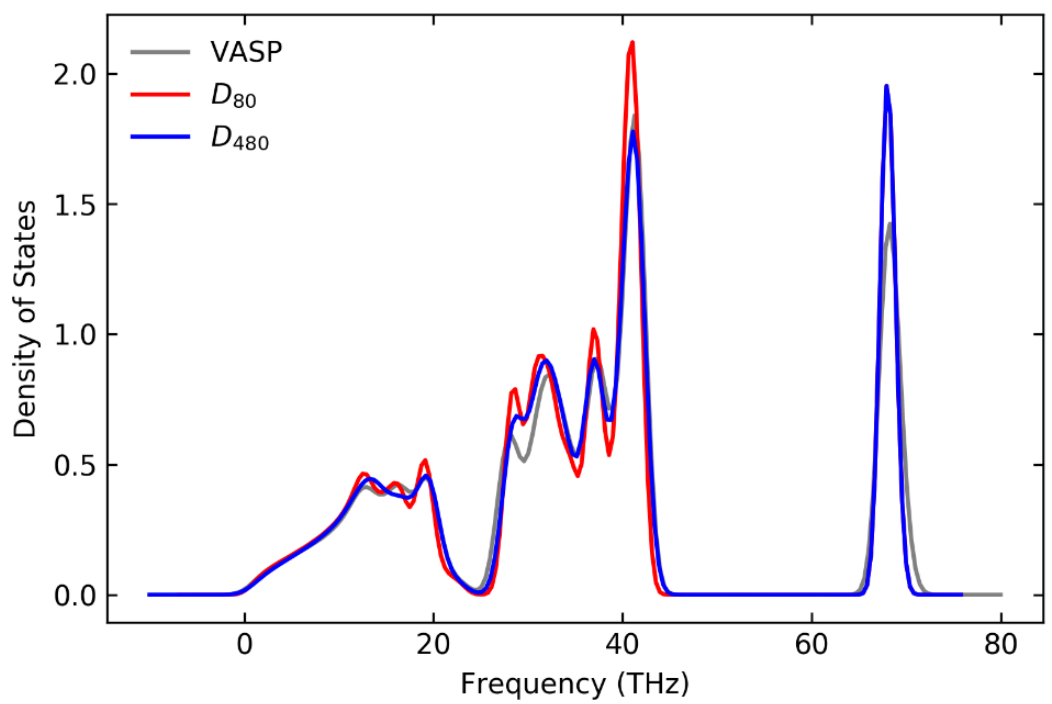

Figure S2: Phonon density of states of graphane at $T=0 \mathrm{~K}$ computed using $D_{80}, D_{480}$ and compared with VASP results. 
in the predictions by the two models. This indicates that training a DP with a smaller system of graphane is sufficient to develop a reliable force field.

\section{S4. Phonon calculations:}

There were two python-based tools used to compute phonon properties: phonopy ${ }^{5}$ for VASP and phonoLAMMPS ${ }^{6}$ for the Large-scale Atomic/Molecular Massively Parallel Simulator (LAMMPS). All phonon bands for a $2 \times 2 \times 1$ supercell of the chair configuration of graphane were first calculated with a consistent Brillouin-zone (BZ) phonon sampling of $33 \times 33 \times 1$ mesh point. The PDOS were then computed from the phonon dispersion curves. The path inside the $\mathrm{BZ}$ is from $\Gamma$ to $\mathrm{M}$ to K. Figure $\mathrm{S} 2$ shows these phonon bands for graphane using VASP, DP and AIREBO $^{7}$.

These phonon dispersion curves were projected onto a phonon density of states plot as shown in Figure 3 of the main text. Similar to the density of states plots, DP predicts the general trend of bands in the three frequency ranges: low, intermediate, and high. AIREBO underpredicts the frequencies of the $\mathrm{C}-\mathrm{H}$ stretching at higher frequencies. It also overpredicts the intermediate $\mathrm{C}-\mathrm{H}$ modes, which were expected to be below by a few THz from $45 \mathrm{THz}$. AIREBO even has one low-frequency mode with some negative character, indicating material instability. 

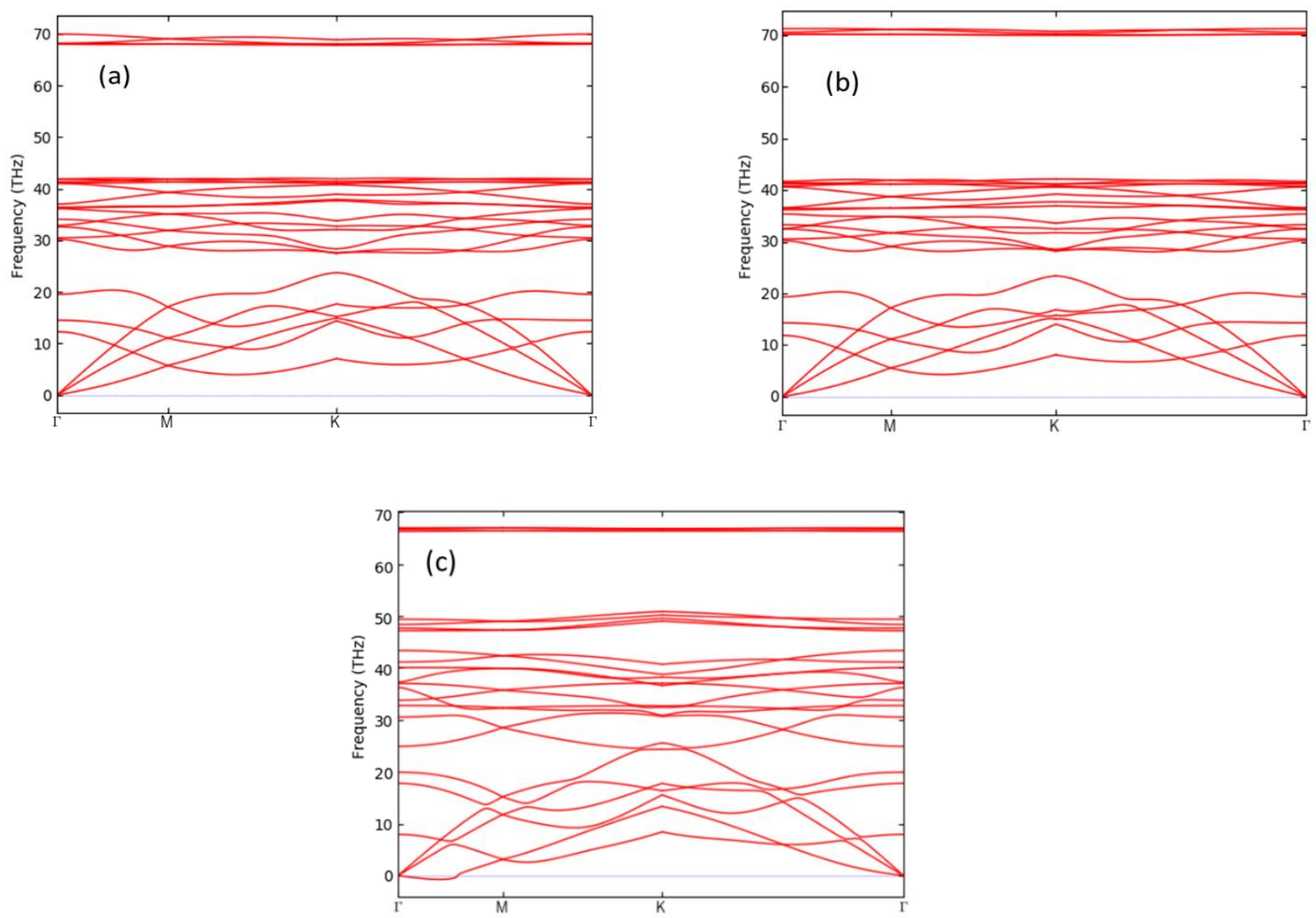

Figure S3: Phonon dispersion spectrum for graphane computed using the quasi-harmonic approximation for (a) VASP, (b) DP and (c) AIREBO using phonopy for (a) and phonoLAMMPS for (b) and (c). 


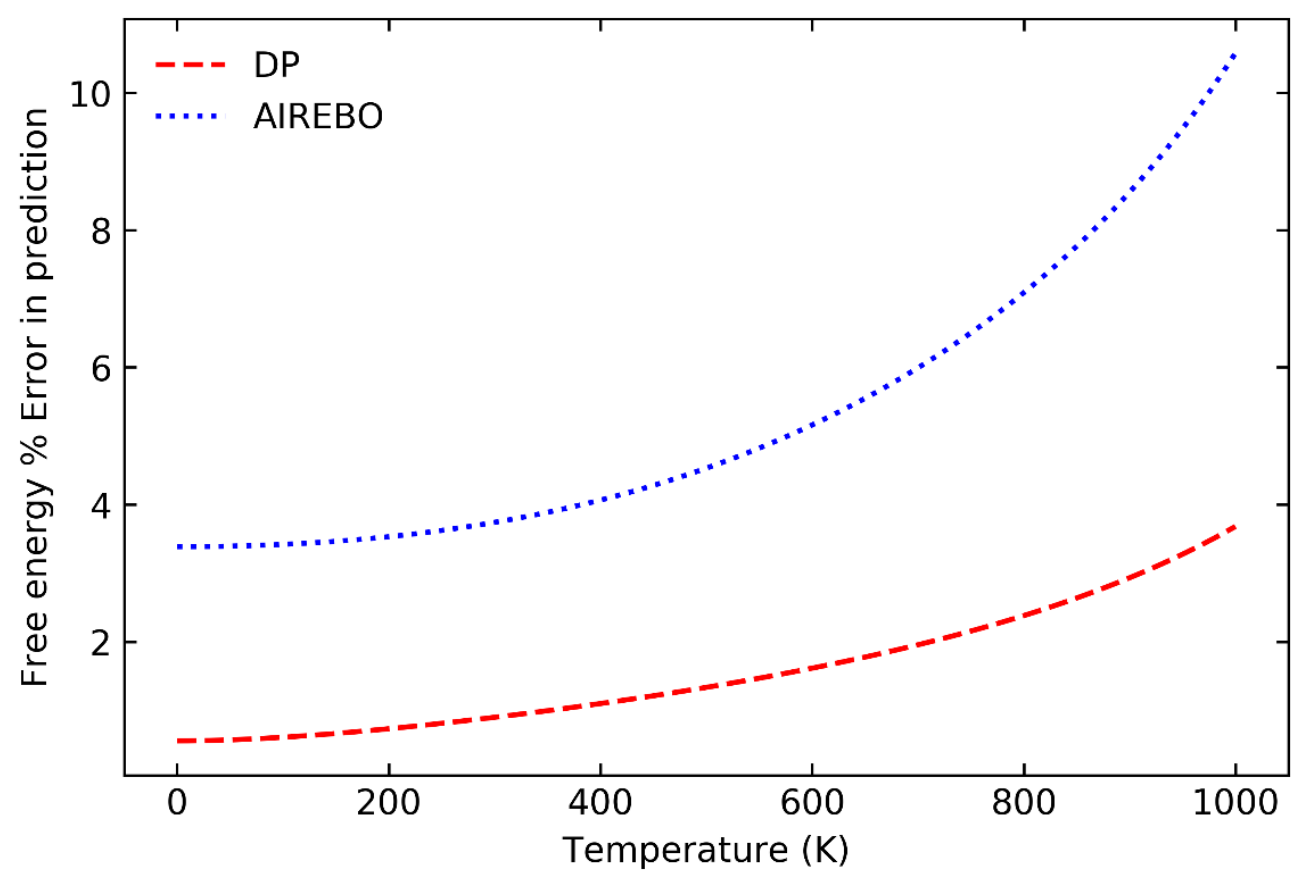

Figure S4: Trend in \% error in prediction of free energy as a function of temperature. The two models used as DP (dashed red) and AIREBO (dotted blue) and compared to VASP.

Table S2: Percentage error in prediction of thermal properties at $1000 \mathrm{~K}$ : free energy (A), heat capacity $\left(\mathrm{C}_{\mathrm{v}}\right)$ and entropy $(\mathrm{S})$ using DP and AIREBO compared to VASP.

\begin{tabular}{llll}
\hline Potential & $\mathbf{A ~ ( k J / m o l )}$ & $\mathbf{C}_{\mathbf{v}}(\mathbf{J} / \mathbf{m o l ~ K})$ & $\mathbf{S}(\mathbf{J} / \mathbf{m o l ~ K})$ \\
\hline DP & $3.7 \%$ & $0.17 \%$ & $2.2 \%$ \\
AIREBO & $10.6 \%$ & $1.8 \%$ & $5.5 \%$ \\
\hline
\end{tabular}




\section{S5. VACF decorrelation}

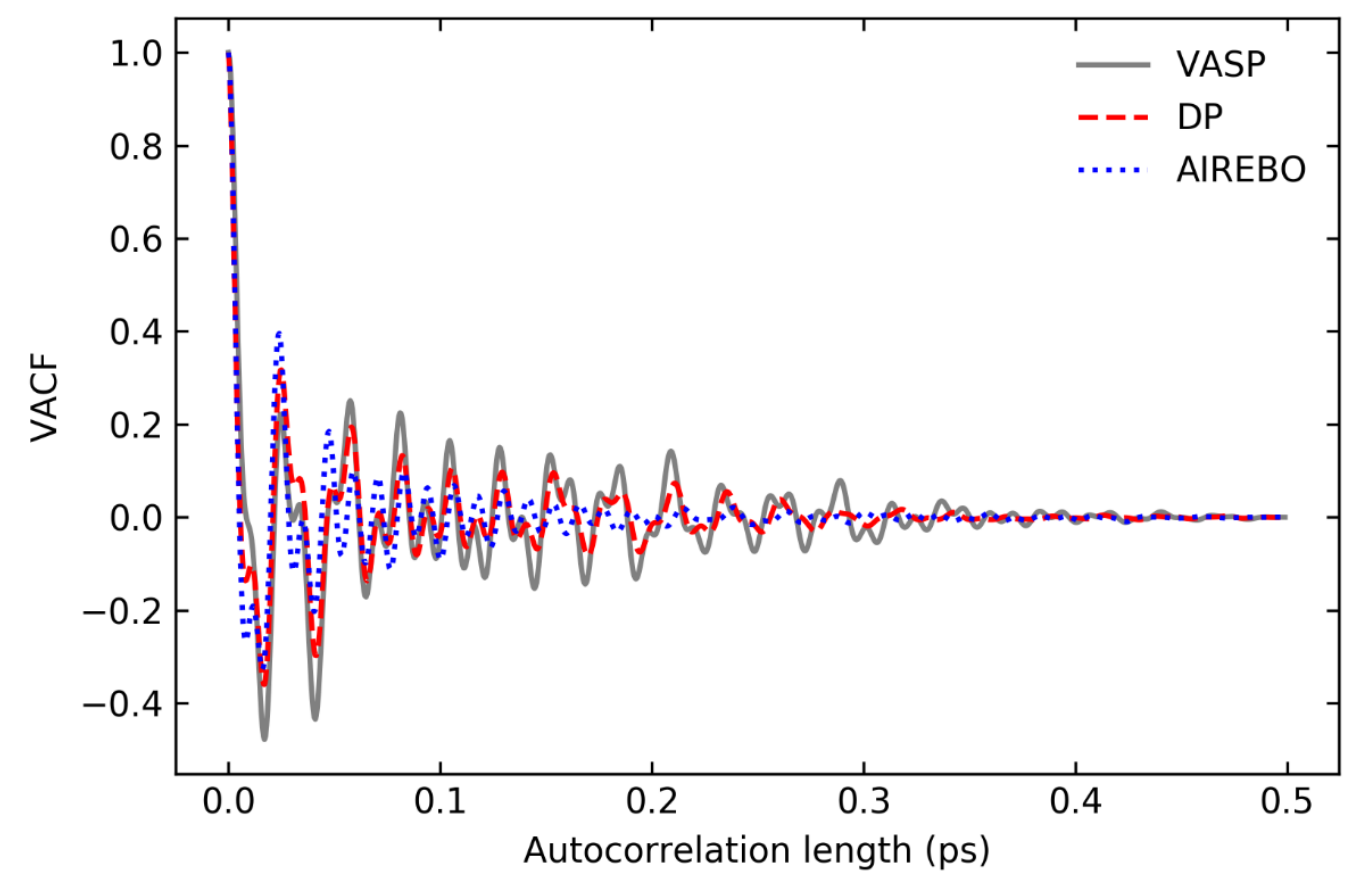

Figure S5: Velocity autocorrelation function computed from MD simulations of graphane at $1000 \mathrm{~K}$ using VASP, DP and AIREBO over the entire autocorrelation length.

\section{S6. Mechanical Properties}

Stress-strain curve

In order to simulate deformation of graphane in LAMMPS, MD simulations were run at a fixed temperature. The graphane system was in an orthorhombic cell with 160 atoms (as shown in Figure 6 in the main text). This system was first equilibrated in a Nosé-Hoover thermostat and barostat $(N p T)$ with a fixed temperature of $300 \mathrm{~K}$ and 0 bar. Upon equilibration, an external linear strain of $0.001 / \mathrm{ps}$ was applied in the $y$ direction from 0 to $0.25 \%$ strain. The stress response upon deformation is plotted for DP and AIREBO in Figure S4. 


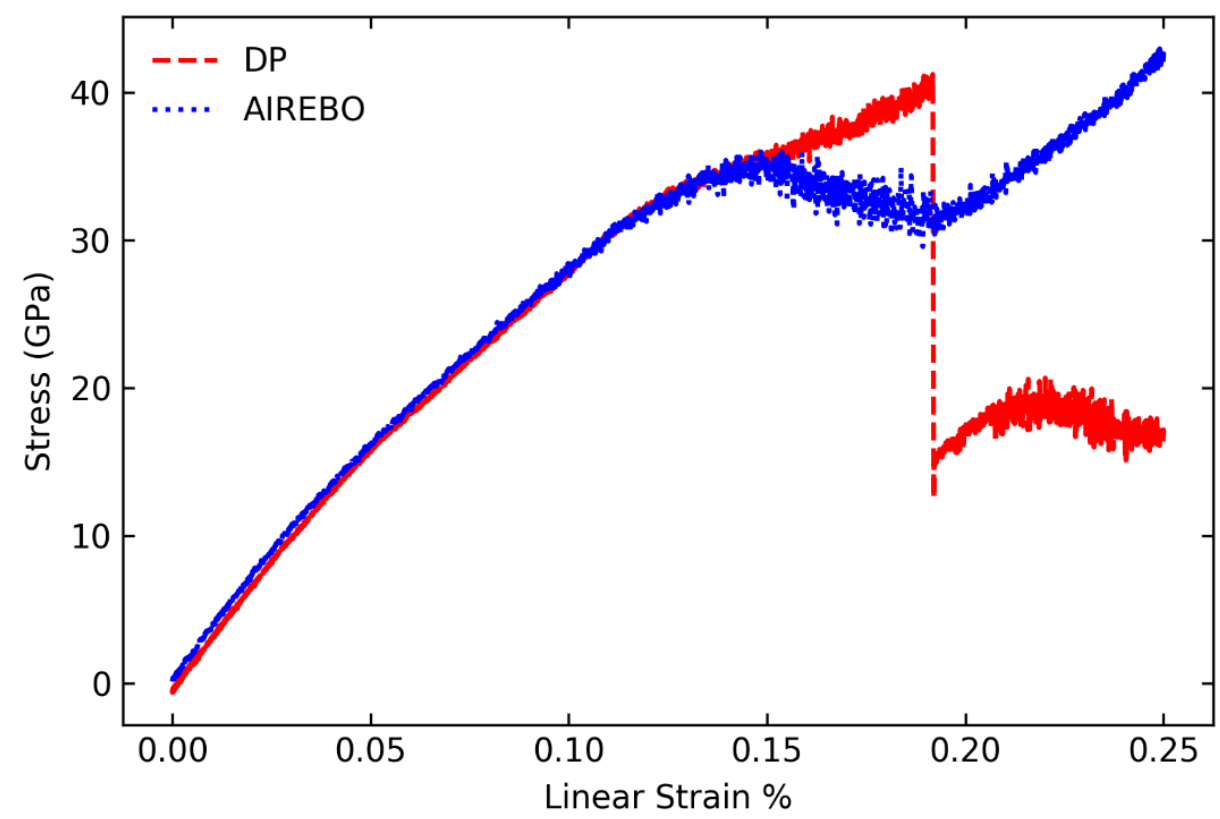

Figure S6: Stress vs linear strain computed using DP and AIRBO for graphane system in Figure 6.

\section{Tensile modulus calculation}

The tensile modulus was calculated from the slope of the linear part of this stress-strain curve. We chose the linear strain region from 0.0 to $0.01 \%$ as the linear stress response and fit a straight line to the simulation data. The slope of this curve corresponds to the tensile modulus of graphane, which gave a value of $354.78 \mathrm{GPa}$. A plot with the fitting is shown in Figure S5. These calculations were performed just using the DP. 


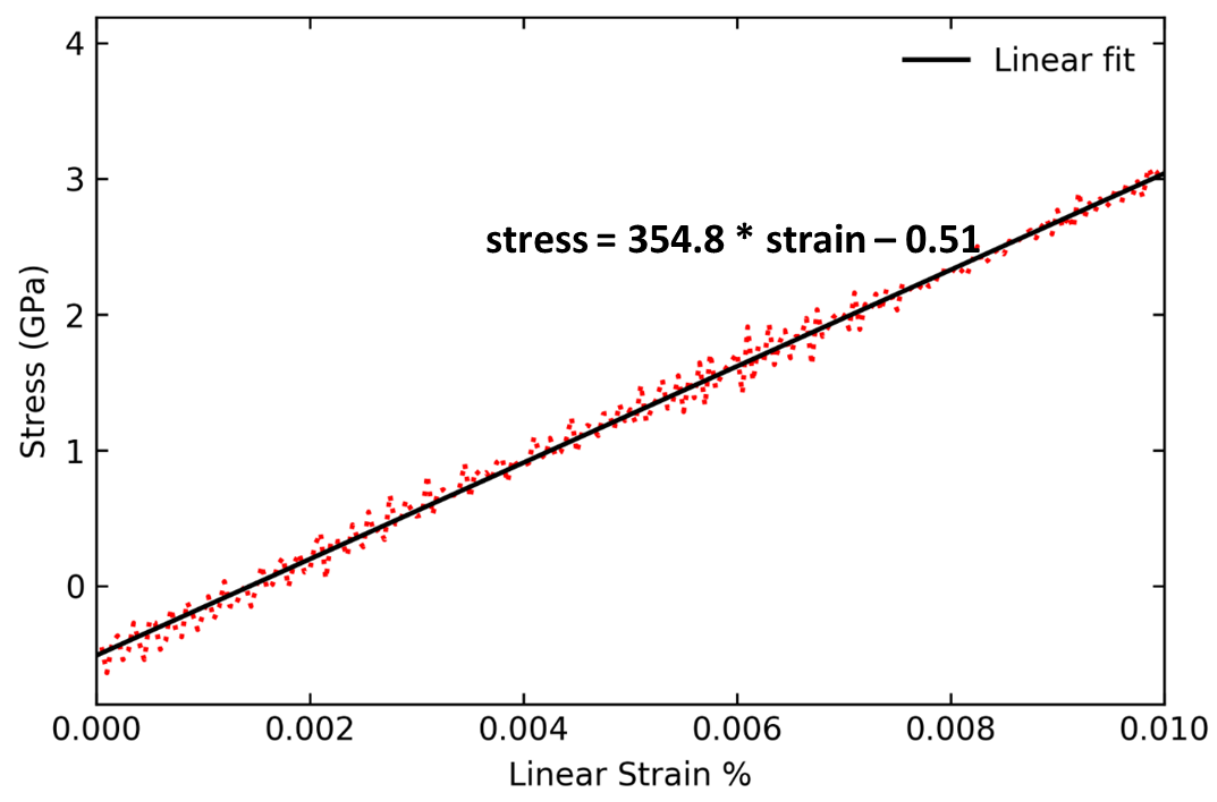

Figure S7: Stress vs linear strain computed using DP from 0.0 to $0.01 \%$ strain. The black line is a linear fit to the data points, the slope of which corresponds to the tensile modulus of graphane. The tensile modulus was found to be $354.8 \mathrm{GPa}$ from the linear fit.

\section{Elastic and plastic regions}

To classify the regions before and after the critical strain (from Figure 8 in the main text) as elastic and plastic respectively, test calculations were performed. Two deformed configurations before and after the critical strain were taken and the systems were relaxed using the DP. The green circle and blue star from Figure S8a were the two configurations picked for relaxation. The lattice constants for the ground state structure before strain was applied (Figure 7) are $25.39 \AA$ and $8.89 \AA$ in the $x$ and $y$ directions, respectively. The strained configuration corresponding to the green dot in Figure S8a has lattice constants of $25.26 \AA$ and $9.88 \AA$ in the $x$ and $y$ directions, respectively. This configuration was relaxed using the DP, with the energy convergence shown as the green curve in Figure S8b. We see that this configuration relaxes to a local minimum having an energy $0.3 \%$ ( $\Delta E=0.02 \mathrm{eV} /$ atom) higher than the ground state energy. The final lattice constants for this relaxation are $8.89 \AA$ in the $y$ direction, which corresponds to the ground state lattice constant, but the $x$ direction lattice constant converges to $22.73 \AA$, which is considerably smaller than the ground state. The top and side views of the relaxed configuration are shown in Figure S9a and b. We see that the system become buckled along the $x$ direction upon relaxation, which accounts for the reduction of the lattice constant in the $x$ direction. We have verified from DFT calculations that the energy difference between the ground state structure and the relaxed structure shown in Figure S9 is indeed about $0.02 \mathrm{eV} /$ atom. This means that the DP gives reliable energies for this configuration. Hence, the strain for the point shown by the green dot in Figure S8 is in the elastic region given that the lattice constant along the 
direction of strain ( $y$ direction) relaxes back to the unstrained value. The buckled structure is an artifact of the way the system relaxes from the strained configuration.

We now consider the structure after the critical strain shown as the red star in Figure S8. The strained lattice constants are $24.92 \AA$ and $10.87 \AA$ in the $x$ and $y$ directions, respectively. Relaxation of this structure with DP converges to a structure having an energy $0.6 \%(\Delta E=$ $-0.04 \mathrm{eV} /$ atom) lower than the ground state, as seen by the blue curve in Figure Sb. The relaxed lattice constants are $22.96 \AA$ and $10.02 \AA$ in the $x$ and $y$ directions, respectively. The relaxed configuration is shown in Figure S9c and d. We see that this structure also exhibits buckling along the $x$ direction. However, the system does not relax back to the unstrained lattice constant in the $y$ direction, but instead converged to a lattice constant that is stretched by about $14 \%$ relative to the ground state. The $\mathrm{C}-\mathrm{C}$ bond distances for this post-critical strain relaxed structure are significantly different from the ground state structure, which has bond lengths of $1.55 \AA$. The relaxed post-critical strain system has a variety of different bond lengths, ranging from values of about $1.47 \AA$ to $1.6 \AA$, depending on the direction of the bond and the location within the supercell. These bond lengths clearly indicate that the configuration corresponding to the blue star in Figure S8a has undergone a plastic deformation. 

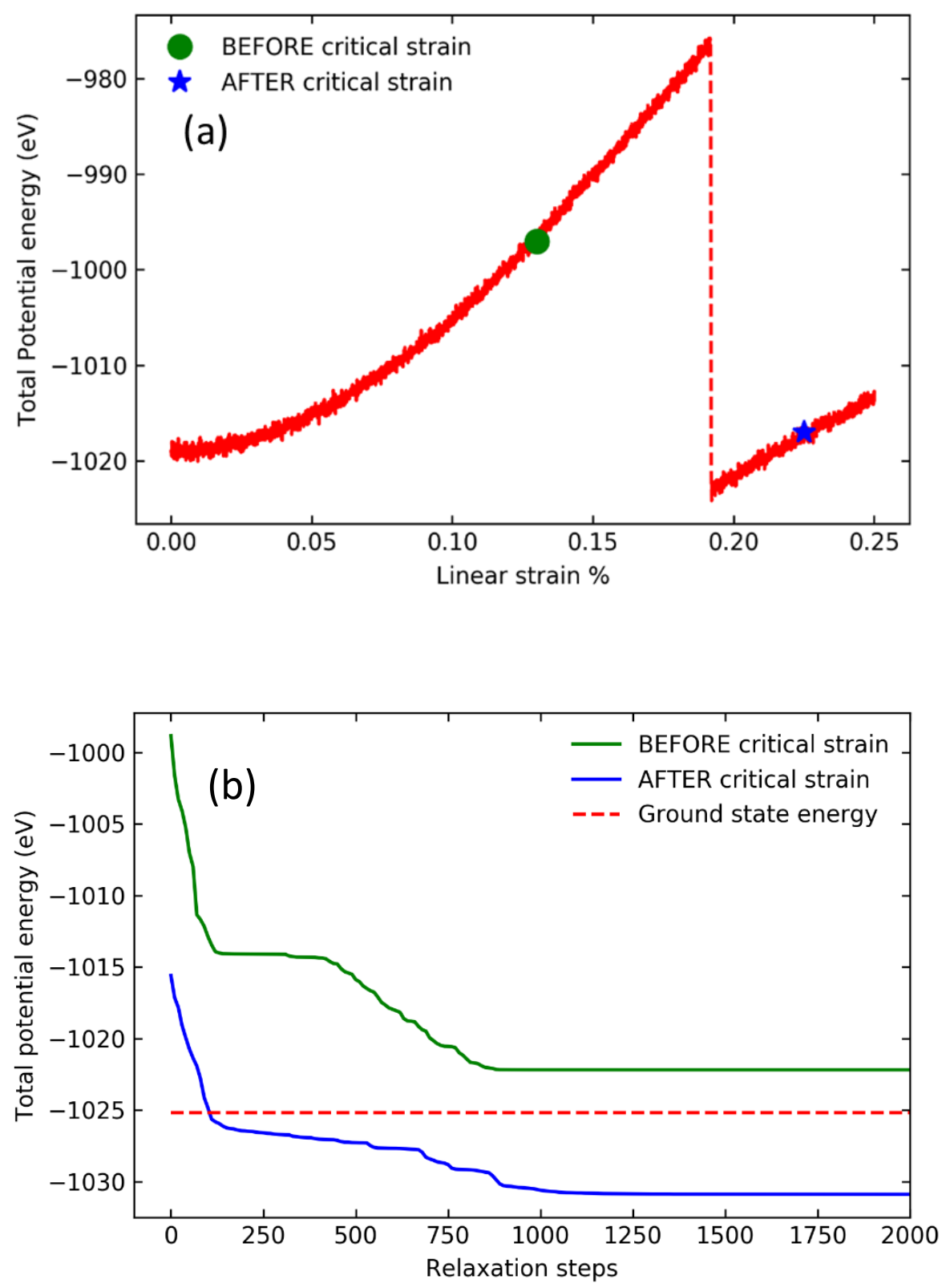

Figure S8: Elastic and plastic region testing: (a) Plot of potential energy of graphane as a function of linear strain (in \%). The green point and blue star were taken from the elastic and plastic regions, respectively. (b) Potential energy plotted for each step in the DP relaxation process. 
(a)

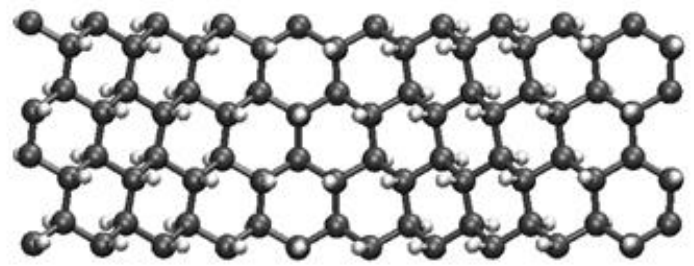

(b)

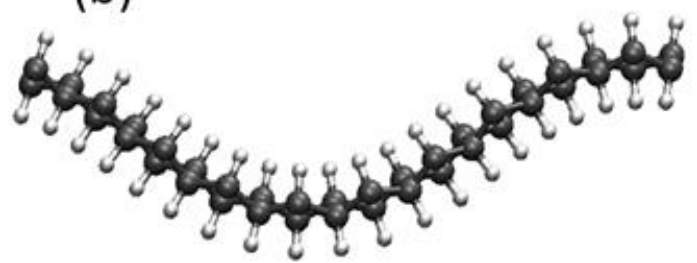

(c)

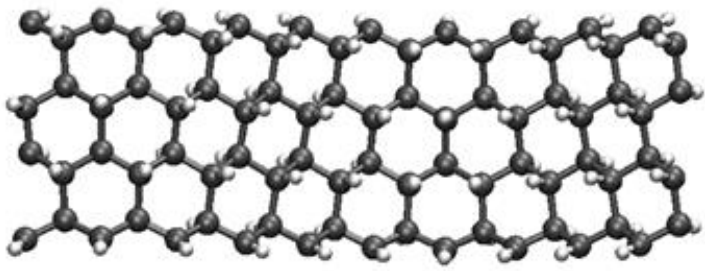

(d)

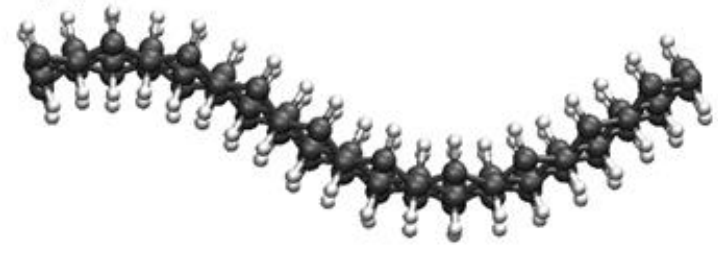

Figure S9: (a) Top ( $x-y$ plane) and (b) side ( $y$-z plane) views of DP relaxed structures starting from a structure before critical strain (green circle in Figure S8a). (c) and (d) are the top and side views of a structure after critical strain (blue star in Figure S8a).

\section{S7. Scaling comparison with fit}

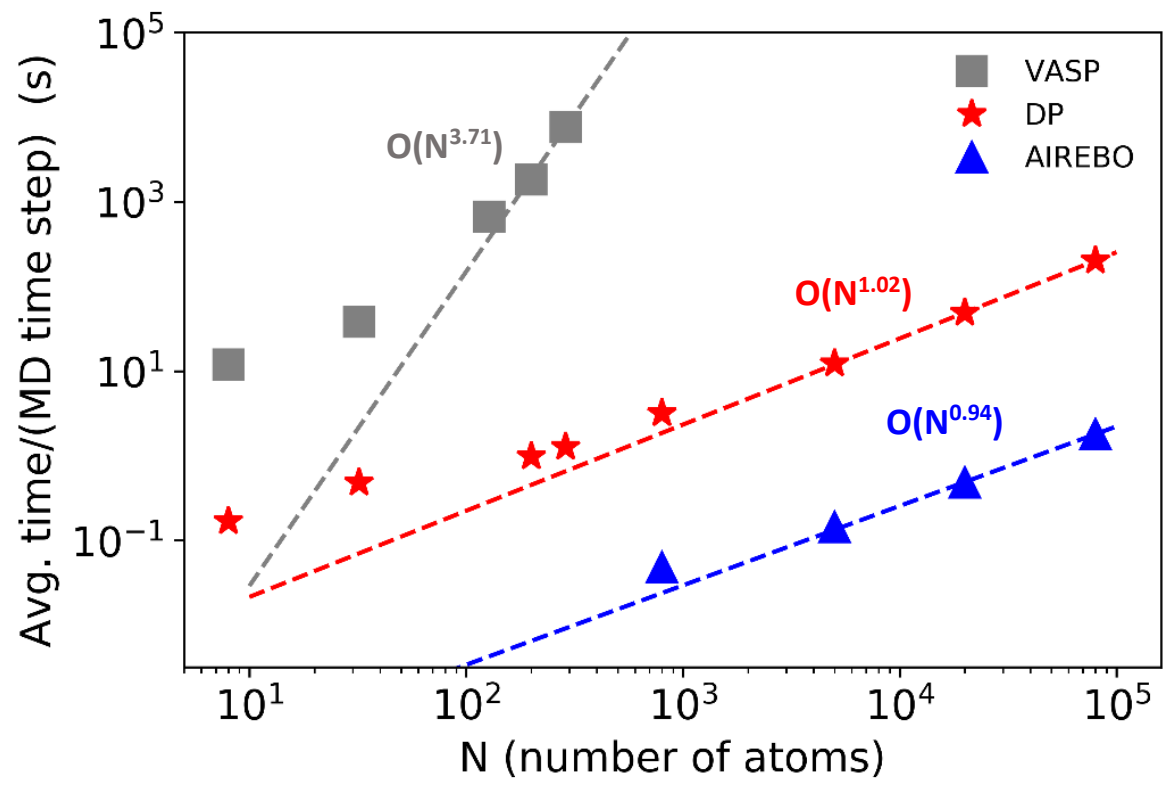

Figure S10: Scaling comparison with fit: VASP, DP and AIREBO was used to compute the average time per MD time step in seconds for different system sizes that have varying number of atoms $(\mathrm{N})$ in graphane. Dashed lines represent the linear fit in log space. VASP scales in $\mathrm{O}\left(\mathrm{N}^{3.71}\right)$, whereas DP and AIREBO scale almost linearly with $\mathrm{O}\left(\mathrm{N}^{1.02}\right)$ and $\mathrm{O}\left(\mathrm{N}^{0.94}\right)$ respectively. 


\section{References}

1. He, K.; Zhang, X.; Ren, S.; Sun, J., Deep Residual Learning for Image Recognition. IEEE Conference on Computer Vision and Pattern Recognition (CVPR); IEEE 2016.

2. $\quad$ Kresse, G.; Furthmüller, J., Efficient Iterative Schemes for Ab Initio Total-Energy Calculations Using a Plane-Wave Basis Set. Phys. Rev. B 1996, 54, 11169-11186.

3. $\quad$ Blöchl, P. E., Projector Augmented-Wave Method. Phys. Rev. B 1994, 50, 17953-17979.

4. Hoover, W. G.; Holian, B. L., Kinetic Moments Method for the Canonical Ensemble Distribution. Phys. Lett. A 1996, 211, 253-257.

5. Togo, A.; Tanaka, I., First Principles Phonon Calculations in Materials Science. Scr. Mater. 2015, $108,1-5$.

6. Carreras, A., LAMMPS interface for phonon calculations using phonopy https://github.com/abelcarreras/phonolammps. 2019.

7. Stuart, S. J.; Tutein, A. B.; Harrison, J. A., A Reactive Potential for Hydrocarbons with Intermolecular Interactions. J. Chem. Phys. 2000, 112, 6472-6486. 